\title{
Geschäftsmodelle mitentwickeln - ein neues Handlungsfeld der Betriebsräte
}

DETLEF GERST

\section{Eine verhängnisvolle Arbeitsteilung}

Wer im Betrieb die Interessen der Beschäftigten vertritt, hat insbesondere das Ziel im Blick, diese bei Veränderungen zu schützen. Betriebsräte tun dies mit Erfolg, indem sie vor allem die Mitbestimmungsrechte zu sozialen Angelegenheiten nutzen, die im Betriebsverfassungsgesetz verankert sind, und indem sie zugleich darauf dringen, möglichst früh über geplante Modernisierungsprozesse informiert zu werden. Darin sehen die meisten Betriebsräte ihre Hauptaufgabe. Deutlich weniger Wert legen sie darauf, sich aktiv an der Entwicklung von Innovationsstrategien und Geschäftsmodellen des Unternehmens zu beteiligen. In aller Regel gilt: Nicht nur die Unternehmensleitung sieht die übergeordnete Unternehmensstrategie und deren Entwicklung allein in ihrer Verantwortung, das sehen auch die meisten Betriebsräte und Vertrauensleute so. Damit gibt es eine Arbeitsteilung, die von beiden Seiten gleichermaßen akzeptiert wird: Das Management entscheidet über die Strategie, der Betriebsrat reguliert die Folgen.

Warum verhalten sich Betriebsräte so? Warum bringen sie sich kaum in die strategische Modernisierung von Betrieben ein? Das hat mehrere Gründe. Der erste: Wer sich da einmischt, bewegt sich rechtlich auf dünnem Eis. Denn das Betriebsverfassungsgesetz bietet dafür nur schwache Beteiligungsrechte, sodass diejenigen, die sich aktiv in das Innovationsgeschehen im Betrieb einbringen, das hohe Risiko eingehen, im Konfliktfall zu unterliegen. Und solche Konflikte könnten zudem eskalieren, wenn Management und Eigentümer für dieses Feld ihr Recht auf unternehmerische Selbstbestimmung reklamieren.

Einen weiteren Grund halte ich jedoch für schwererwiegend: Betriebsräte, die auf dem Feld der Unternehmensstrategie aktiv sind, gelten gemeinhin als Co-Manager. Und die Strategie des Co-Managements hat in Teilen der Gewerkschaften immer noch einen schlechten Ruf. Wer sich gemeinsam mit dem Management engagiert, um Produktpaletten und Herstellungsprozesse zu modernisieren, setzt sich als Betriebsrat auch heute noch dem Ver- dacht aus, im Zweifel dem Management näher zu stehen als den Beschäftigten. Zwar haben Betriebsräte mit der gewerkschaftlichen Strategie „Besser statt billiger“ in ausgewählten Betrieben gute Erfahrungen gemacht, gelang es ihnen doch damit, eine nachhaltige betriebliche Innovationspolitik einzufordern. ${ }^{1}$ Auch wegen dieser Erfahrungen ist die Sicht auf die Strategie des Co-Managements heute weniger ideologisch geprägt als zuvor. Dennoch hat sich das Selbstverständnis vieler Betriebsräte noch nicht grundlegend geändert.

Betriebsstrategien mit zu entwickeln ist wichtiger denn je. Das zeigt der 2019 erstellte Transformationsatlas der IG Metall. ${ }^{2}$ Basierend auf den Befragungen von Betriebsräten in mehr als 2000 Betrieben zeigt sich hier ein wichtiges Ergebnis: Etwa die Hälfte der Unternehmen kümmert sich unzureichend um ihre strategische Ausrichtung und damit um ihre Zukunft - ein großes Defizit, das erneut die Frage aufwirft, ob das Thema Strategieentwicklung wirklich allein der Arbeitgeberseite überlassen werden darf. Deshalb diskutiere ich im Folgenden genauer die Auswertung der Befragung.

\section{Ohne wirksame Betriebsstrategie keine gute Arbeit}

Es war schon immer fragwürdig, wenn sich die Interessenvertretung kaum um die betriebliche Strategieentwicklung kümmerte, denn dabei geht es immer auch um

1 Lehndorff, S./ Haipeter, T./ Bromberg, T. / Brettschneider, A (2010): Konzepte gegen die Krise? Chancen und Ambivalenzen betrieblicher "Besser"-Strategien für Arbeitspolitik und Interessenvertretungen, in: WSI-Mitteilungen 63 (9), S. 451-457, https://www.wsi.de/de/wsi-mitteilungen-kon zepte-gegen-die-krise-chancen-und-ambivalenzen-betrieb licher-aposbesserapos-strategien-12721.htm

2 IG Metall (2019): Digitale Pressemappe zum Transformationsatlas, verfügbar unter https://www.igmetall.de/presse/ pressemitteilungen/hofmann-pressekonferenz-transforma tionsatlas 
Anzahl und Qualität von Arbeitsplätzen. Ein Beispiel: Betriebe, die es versäumen, mit innovativen Produkten und Dienstleistungen rechtzeitig neue Märkte zu erschließen und deren Herstellung mit neuester Technik produktiv und flexibel zu gestalten, befinden sich in einer Strategiekrise. Als Folge wird mittel- und langfristig der Umsatz sinken, weshalb Arbeitsplätze gefährdet werden und auch zunehmend die finanziellen Mittel fehlen, um in deren ergonomische Gestaltung und in die Qualifikation der Belegschaft zu investieren. Damit aber drohen auf Dauer, Gesundheit, Kompetenz und Motivation der Belegschaft wie auch die Attraktivität des Arbeitgebers zu schwinden; dem Unternehmen wird es dann immer schwerer fallen, neues Fachpersonal zu gewinnen. Das ist kein unbedachtes Krisengemälde, sondern das sind begründete Annahmen und Erfahrungen. So führen Defizite in der Betriebsstrategie zumindest langfristig in Abwärtsspiralen, die immer schwieriger zu stoppen sind. Oft bleibt solchen Betrieben als Option nur noch der defensive pure Kostenwettbewerb, der in der Regel auf dem Rücken der Belegschaft ausgetragen wird - und der angesichts der internationalen Konkurrenz sowieso in fast allen Fällen erfolglos bleibt.

Die Konsequenz für Betriebsräte und Gewerkschaften: Kümmern sich Betriebe nur nachlässig und mangelhaft um ihre Strategie, dann entsteht daraus für die Interessenvertretungen geradezu eine Pflicht, sich aktiv mit diesem Thema zu befassen. Denn ohne eine hochwertige strategische Planung werden Betriebsräte wiederum kaum in der Lage sein, die Beschäftigten vor unsicherer Beschäftigung, materiellen Verlusten und wachsender Arbeitsintensität zu schützen. Die entscheidende Konsequenz aus den bisherigen Überlegungen: Ich gehe davon aus, dass Betriebsräte heute nur dann ihre Schutzfunktion auf Dauer wirksam wahrnehmen können, wenn sie Management und Eigentümer drängen, sich mit hoher Energie und Kompetenzen um die Betriebsstrategie zu kümmern und wenn sie sich selbst auch an dieser Arbeit beteiligen. Das wird sicher nicht in allen Betrieben möglich sein. Aber: Die Strategieentwicklung auch heute noch als eigenes Handlungsfeld der Interessenvertretung grundsätzlich abzulehnen käme einem schweren Fehler gleich. Bedeutete dies doch, fundamentale Voraussetzungen nicht mitgestalten zu wollen, die unverzichtbar sind, um im Betrieb „Gute Arbeit“ durchzusetzen. Zur Erinnerung: Es geht dabei gemäß den Kriterien des DGB-Index Gute Arbeit $^{3}$ - um die drei Dimensionen Arbeitsbelastungen, Ressourcen für den Umgang mit Belastungen sowie Sicherung von Arbeit und materieller Existenz.

Ohne eine strategische Unternehmensentwicklung ist also eine qualifizierte Arbeitspolitik kaum vorstellbar. Und: Das Gewicht dieser Planung nimmt heute noch einmal zu. Der Grund dafür ist eine Transformation von Wirtschaft und Arbeit, die sich beschleunigt und vielfältig äußert. Es geht um Digitalisierung und die mit ihr verbundene, komplexer werdende Vernetzung, um den wachsenden Stellenwert klimafreundlicher Produkte und entsprechender Herstellungsprozesse. Strategische Planung wird zudem anspruchsvoller: Früher war sie bereits dann erfolgreich, wenn Betriebe aus der Beobachtung von Märkten und Wettbewerbern ihre Stärken und Schwächen erkannten und ihre Angebote und Prozesse entsprechend modernisierten; allzu weit mussten sie sich dabei von ihren etablierten Märkten und Angeboten nicht entfernen. Heute geht es um viel mehr: nämlich um die Entwicklung neuer Geschäftsmodelle und damit um die Fähigkeit, sich als Unternehmen von Zeit zu Zeit neu zu erfinden.

Wer neue Geschäftsmodelle entwickeln will, muss nach Gassmann et al. diese vier Fragen zumindest in Teilen neu beantworten: ${ }^{4}$ Was bieten wir den Kunden an? Wer sind unsere Zielkunden? Wie stellen wir die Leistung her? Und: Wie wird der Wert erzielt? Den Autoren zufolge verlangt ein innovatives Geschäftsmodell in mindestens zwei dieser Dimensionen eine Veränderung. Bei der Entwicklung neuer Geschäftsmodelle gelten heute vor allem die Möglichkeiten der Vernetzung als aussichtsreich, welche die Digitalisierung bietet. Konkret: Digitale Plattformen und Business Eco Systeme sind zu nutzen oder für die eigenen Geschäfte gar selbst zu entwerfen.

Neue Geschäftsmodelle zu entwickeln ist also zu einer vorrangigen unternehmerischen Aufgabe geworden. Dies bedeutet auch: Die Entwicklung von Geschäftsmodellen muss der Produkt- und Prozessinnovation vorausgehen. In der Praxis liegen jedoch genau hier die Schwachstellen: „Während in den meisten Unternehmen etablierte Prozesse zur Entwicklung neuer Produkte bestehen, fehlen solche Vorgehensmodelle zur systematischen Entwicklung neuer Geschäftsmodelle häufig. “ ${ }^{5}$

\section{Befunde des IG-Metall-Transformationsatlasses}

\section{Datengrundlage}

Die folgenden Auswertungen beruhen auf dem im Jahr 2019 erstellten Transformationsatlas der IG Metall. Grundlage waren betriebliche Workshops zu Stand und Problemen der Transformation. Abgehalten wurden sie zwischen April und Dezember 2019 in insgesamt 2057 Betrieben. In jedem Betrieb bearbeitete eine jeweils vom Betriebsrat zusammengestellte Fachgruppe einen aus 143 Fragen bestehenden Untersuchungsbogen. Der Transformationsatlas ermittelt damit repräsentativ die Lage in Betrieben, in denen insgesamt rund 1,7 Mio. Arbeitnehmerinnen und Arbeitnehmer der Branchen Metall und Elektro, Holz und

3 DGB-Index Gute Arbeit (2019): Jahresreport 2019. Arbeit am Limit. Themenschwerpunkt Arbeitsintensität.

4 Gassmann, O./Frankenberger, K./Csik, M. (2017): Geschäftsmodelle entwickeln. 55 innovative Konzepte mit dem St. Galler Business Modell Navigator, München, S. 7.

5 VDI / VDE (2016): Digitale Chancen und Bedrohungen - Geschäftsmodelle für Industrie 4.0, S.17. 
Kunststoff sowie Textil und Bekleidung beschäftigt sind. Gegenstand der Untersuchung waren unter anderem: Verlagerung von Produktion und Entwicklung, Digitalisierung und Dekarbonisierung. Mit der Untersuchung wurde ermittelt, mit welchen Trends die Betriebe konfrontiert sind, welche Entwicklungen für Arbeit und Beschäftigung $\mathrm{zu}$ erwarten sind und wie gut Unternehmensleitungen und Betriebsräte auf den Wandel vorbereitet sind. Die Workshops hatten neben der Gewinnung von Daten das Ziel, unter den Betriebsräten und Betriebsbetreuer*innen der IG Metall eine erste gründlichere Debatte über die Transformation anzustoßen.

\section{Starker Anpassungsdruck}

Der Transformationsatlas zeigt vor allem: Ein Großteil der Betriebe unterliegt einem starken Anpassungsdruck; die Probleme unterscheiden sich jedoch wesentlich von Betrieb zu Betrieb. Die künftige Umsatzentwicklung schätzen die befragten Betriebsräte so ein: In $25 \%$ der Betriebe werden die Umsätze sinken, in $24 \%$ gleichbleiben und in $43 \%$ werden sie zunehmen. Die Beschäftigung wird in $33 \%$ der Betriebe abnehmen, in $38 \%$ stabil bleiben und in $26 \%$ zunehmen. ${ }^{6}$

Um die Beschäftigung zu sichern, ist es notwendig, dass die Betriebe ausreichend über marktfähige Produkte und Dienstleistungen verfügen, die sie auch stets modernisieren. Die Betriebsräte von insgesamt $29 \%$ der Betriebe gehen allerdings davon aus, dass es diesen nicht gelingen wird, „durch die Entwicklung neuer Produkte das Auslaufen alter Produkte in den nächsten drei Jahren auszugleichen“. In $24 \%$ der Betriebe denken die Betriebsräte, dies werde teilweise gelingen, und lediglich in $29 \%$ hatten die Interessenvertretungen eine optimistische Erwartung. ${ }^{7}$

Damit liegt die entscheidende Aufgabe auf der Hand: In Betrieben mit negativer und skeptischer Prognose ist es dringend geboten, an einer zukunftsträchtigen wirksamen Betriebsstrategie zu arbeiten. Aber auch dort, wo die Prognose eher optimistisch ausfällt, darf diese Arbeit nicht vernachlässigt werden. Der Grund: Ändert sich die Umwelt der Betriebe, können selbst Strategien in eine Sackgasse führen, die über viele Jahre Erfolge brachten. Ändern kann sich vieles und das oft rasch: beispielsweise Erwartungen und Lebensgewohnheiten der Kunden, neue Techniken sind verfügbar, es gibt neue Partner und Investoren, die Strategien und Angebote der Wettbewerber ändern sich. Strategieentwicklung bedeutet also auch, diese Umwelt stets im Auge zu behalten und zu analysieren, ob der Betrieb ausreichend Chancen nutzt und Risiken vermeidet, ob er rechtzeitig die notwendigen Anpassungen vornimmt. Und zur Strategieentwicklung zählt, vorab neue Geschäftsmodelle, Techniken und Produkte zu erproben und erst dann über deren Umsetzung zu entscheiden.

Der Anpassungsdruck drückt sich auch in den Berichten der Betriebsräte über starke Veränderungstreiber aus. Auf Platz eins steht die „Digitalisierung von Produktion und Prozessen“. In 67\% der Betriebe sehen Betriebsräte darin den künftigen Schwerpunkt der Transformation. Auf Platz zwei steht die Verlagerung „von Produktion, Dienstleistung und Entwicklung"; in 33\% der Betriebe ist das für Betriebsräte der Kern der Veränderung. Mit dem Trend zur Elektromobilität sind nach deren Einschätzung $29 \%$ der Betriebe stark konfrontiert, $18 \%$ sind von der Dekarbonisierung in der Energiewirtschaft und $17 \%$ der Betriebe von der Dekarbonisierung in der Stahlerzeugung sowie der Notwendigkeit zu energieeffizienteren Prozessen jeweils stark betroffen.

\section{Riskantes Defizit:}

\section{Betriebe und ihre ungeliebte Strategiearbeit}

Die Befragung zeigt, es gibt sehr wohl Beispiele für eine vorbildliche strategische Unternehmensentwicklung. Jedoch sehen angesichts der „aktuell praktizierten strategischen Bearbeitung der Transformation im Betrieb“ die Betriebsräte in $36 \%$ der Betriebe - wenn sie Risiken und Chancen, Stärken und Defizite bilanzieren - eher Risiken. In $49 \%$ der Betriebe urteilen die Betriebsräte unentschieden. Nur in $15 \%$ der Betriebe überwiegen nach ihrer Einschätzung eindeutig die Chancen und die Stärken.

Wie begründen die Betriebsräte ihre jeweilige Bilanz? Aufschluss geben die Befragungsergebnisse zu den folgenden Teilaspekten der Strategieentwicklung (Abbildung 1):

Die Ergebnisse zeigen: Die Qualität der strategischen Unternehmensentwicklung ist sehr unterschiedlich. Etwa

\section{ABBILDUNG 1}

\section{Vorbereitungen der Betriebe auf die Transformation ${ }^{A}$}
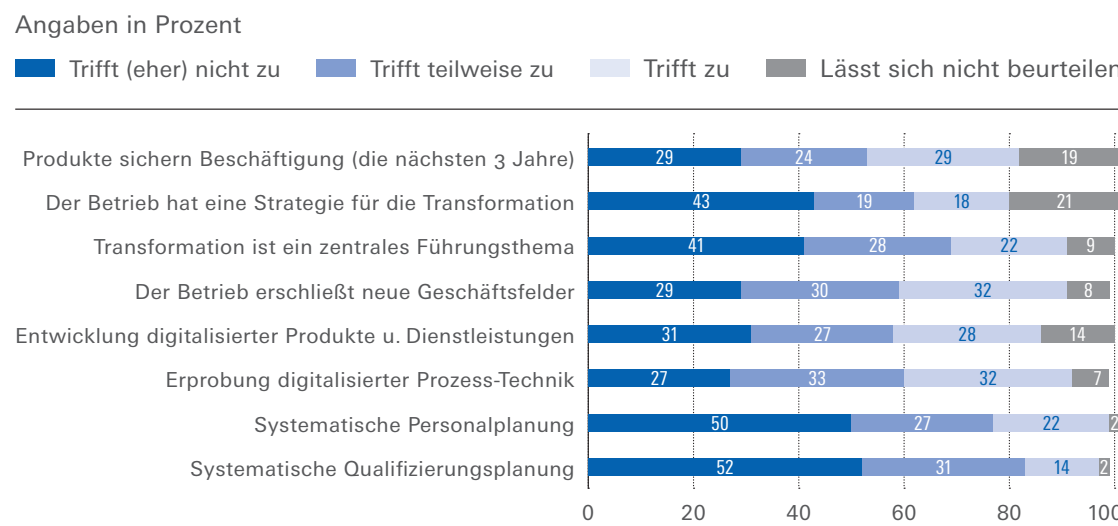

A Die Fragestellung lautete „Wie bereitet sich euer Betrieb/Unternehmen auf die Transformation vor?" Basis der Befragung sind 2057 Betriebe der Metall- und Elektro-, Holz- und Kunststoffsowie Textil- und Bekleidungsindustrie

Quelle: Darstellung des Autors auf Basis der Daten des IG-Metall-Transformationsatlas, siehe Fußnote 2

6 Die Zahlen spiegeln die Situation Ende 2019 wider. Sie dürften sich heute angesichts der Corona-Krise erheblich verändert haben.

7 Auch diese Zahlen dürften sich heute vor dem Hintergrund der Corona-Krise deutlich verändert haben. 


\section{ABBILDUNG 2}

\section{Betriebsratsarbeit im Prozess der betrieblichen Transformation ${ }^{\mathrm{A}}$}

Angaben in Prozent

Trifft (eher) nicht zu

Trifft teilweise zu

Trifft zu

Der Betriebsrat wird über Veränderungsprojekte frühzeitig informiert

Der Betriebsrat ist in Projektentwicklungen und deren Umsetzung einbezogen

Die Belegschaft ist ausreichend informiert, welche Veränderungen im Betrieb in den nächsten Jahren auf sie zukommt

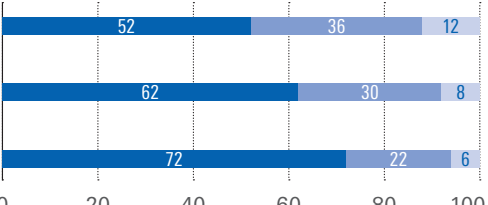

Die Fragestellung lautete „Inwieweit treffen folgende Aussagen zur Betriebsratsarbeit im Prozess der Transformation zu?"

Basis der Befragung sind 2057 Betriebe der Metall- und Elektro-, Holz- und Kunststoffsowie Textil- und Bekleidungsindustrie

Quelle: Darstellung des Autors auf Basis der Daten des IG-Metall-Transformationsatlas, siehe Fußnote 2 nicht abschätzen können, welches Personal und welche Kompetenzen er in den kommenden Monaten und Jahren benötigt.

Betriebe vernachlässigen es, ihre Geschäftsmodelle weiterzuentwickeln. Aber warum? Der Verband VDI/ VDE schöpft Antworten aus seinen praktischen Erfahrungen: ${ }^{8}$

1 Es fehlt das Bewusstsein, dass auch erfolgreich praktizierte Strategien nicht auf Dauer funktionieren.

2 Die Entwicklung von Geschäftsmodellen gehört in vielen Betrieben nicht zu den regelmäßigen Aufgaben.

3 Zudem fehlt es in vielen Betrieben ,an personellen Ressourcen sowie technischer und methodischer Kenntnis“. Diese Einschätzung teilen auch Betriebsräte.

\section{Ohne Betriebsrat und Beschäftigte}

\section{keine gelungene Transformation}

Entscheidet sich ein Unternehmen, an seiner künftigen Strategie zu arbeiten, ist es für alle Beteiligten von Vorteil, die Beschäftigten und deren Interessenvertretung zur Mitarbeit einzuladen, also zu kooperieren. Schließlich wissen die Beschäftigten sehr viel über die Produktionsprozesse. Sollen neue digitale Arbeitsmittel eingeführt werden, dann wird deren Gebrauchstauglichkeit sicher höher sein, wenn ihr Einsatz zuvor gemeinsam mit den Beschäftigten geplant und gestaltet wird. Und: Eine Modernisierung kann im Betrieb auf Dauer sowieso nur gelingen, wenn sie von den Beschäftigten akzeptiert und mitgetragen wird. Die Interessenvertretung wird dabei immer eine zentrale Rolle spielen, genießt sie in der Belegschaft doch fast immer ein besonderes Vertrauen.

Also müssten Management und Eigentümer auch ein Interesse daran haben, Beschäftigte und Betriebsräte an Transformation und Modernisierung aktiv zu beteiligen. Der Transformationsatlas der IG Metall legt jedoch offen, dass es diesbezüglich in den meisten Betrieben erhebliche Defizite gibt (Abbildung 2). In mehr als der Hälfte der Betriebe wird der Betriebsrat nicht früh genug über Veränderungsprojekte informiert. Und in über $60 \%$ der Betriebe ist er an entsprechenden Projekten, deren Entwicklung und Umsetzung gar nicht beteiligt. Damit verstoßen die Unternehmensleitungen nicht nur gegen die Grundgedanken von Mitbestimmung, Kooperation und Sozialpartnerschaft, sie verzichten fahrlässig zum Nachteil des gesamten Unternehmens auch auf wertvolle Ressourcen. Stattdessen lamentieren sie wahrheitswidrig in Medien und auf Konferenzen von Zeit zu Zeit, wie sehr Mitbestimmung die betriebliche Modernisierung verzögere. Richtig ist vielmehr: Wer die Interessenvertretungen zügig, fair und vollständig einbezieht, der beschleunigt in der Regel die Modernisierung, erhöht deren Qualität und fördert deren Akzeptanz und Gelingen. Dass sich Be-

8 VDI/VDE (2016), a.a. O., S. $32 \mathrm{f}$
Die Befragungsergebnisse und Beispiele zeigen: Von der Entwicklung einer schlüssigen und wirksamen Strategie hängt die Zukunft eines Unternehmens ab. Ohne sie kann beispielsweise nicht einmal zielgenau der Bedarf an Personal und Qualifizierung geplant werden. Konkret: Wenn ein Betrieb keine Vorstellung davon hat, was er wie in drei oder fünf Jahren produzieren wird, dann wird er 
triebsräte bezogen auf die Managementkonzepte nicht als „Durchwinkinstanz“verstehen, das verlangt nicht nur das Betriebsverfassungsgesetz, sondern das verlangt zu Recht auch die Belegschaft. Und eine wirkliche Beteiligung der Betriebsräte dient zudem der Qualität und Effektivität der Modernisierung.

Aus den eben angestellten Überlegungen folgt: Unternehmen, die ihre Interessenvertretung nicht an der Strategiearbeit beteiligen, schwächen sich selbst. Sie lassen einen Fundus an Kompetenzen und eine potenzielle Quelle an Legitimation brachliegen. Insofern offenbart die NichtBeteiligung der Betriebsräte ein Gestaltungsdefizit aufseiten der Geschäftsführung. Dass in über 79\% der Betriebe die Beschäftigten nicht einmal ausreichend über bevorstehende Veränderungen im Betrieb informiert, geschweige denn daran beteiligt werden, verstärkt meine Einschätzung noch. Ein erschütternd hoher Teil an Betrieben will offensichtlich nicht nur ohne Masterplan in die Zukunft irren, sondern auch ohne mit Interessenvertretung und Beschäftigten nur ein Wort darüber zu wechseln.

\section{Konsequenz: Sich einmischen wird Pflicht}

Aus den dargelegten Befunden sollten die Gewerkschaften meines Erachtens folgende Konsequenzen ziehen: die Betriebsräte zu ermuntern, sich in die betriebliche Strategieentwicklung einzumischen. Strategieentwicklung allein den Unternehmensleitungen zu überlassen ist mit zu großen Risiken für die Sicherheit der Arbeit und die Qualität der Arbeitsbedingungen verbunden. Es ist wiederum auch Aufgabe der Betriebsräte, in diesem Zusammenhang das Know-how der Belegschaft zu mobilisieren. Dies zu tun entspricht dem Auftrag aus dem Betriebsverfassungsgesetz, wonach die Zukunft von Beschäftigung und gute Arbeitsbedingungen betriebspartnerschaftlich zu sichern sind.

Offensichtlich ist ein Großteil der Betriebsräte auch bereit, sich stärker mit dem Thema Transformation auseinanderzusetzen. $75 \%$ der Betriebsräte wollen sich laut Befragung bei den Herausforderungen der Transformation beraten lassen und sich dafür qualifizieren. Bislang konnte nur in $5 \%$ der Betriebe der Betriebsrat „eine Betriebsvereinbarung zu Digitalisierung, Industrie 4.0 und Transformation abschließen" - eine dramatisch niedrige Zahl. Damit sich dies ändert, wird die IG Metall unter anderem haupt- und ehrenamtliche Prozessbegleiter ausbilden und konkret unterstützen. Im Projekt „Die IG Metall vom Betrieb aus denken" wird die IG Metall bereits in diesem Jahr „Veränderungspromotor*innen“ schulen und mit Coaching unterstützen. Die Erfahrungen der Promotor ${ }^{\star}$ innen werden wiederum genutzt, um die IG Metall strategisch zu modernisieren, ihre Angebote und Ressourcen also stärker auf die betriebliche Transformation auszurichten.

Dass es sich für die Beschäftigten wie für den Betrieb lohnt, Betriebsräte konkret einzubeziehen, das zeigen die in Abbildung 3 präsentierten Befunde.

\section{ABBILDUNG 3}

\section{Betriebe mit und ohne Beteiligung der Betriebsräte an Modernisierungsprojekten ${ }^{A}$}

Angaben in Prozent

Trifft $z u^{B}$

Trifft nicht $z u^{B}$

Der Betriebsrat ist in Projektentwicklungen und deren Umsetzung eingebunden Produkte sichern Beschäftigung (die nächsten 3 Jahre) Betrieb hat eine Strategie für die Transformation Entwicklung digitalisierter Produkte und Dienstleistungen Erprobung digitalisieter Prozess-Technik Systematische Personalplanung Systematische Qualifizierungsplanung Beschäftigte an Arbeitsgestaltung beteiligt Gefährdungsbeurteilungen werden durchgeführt

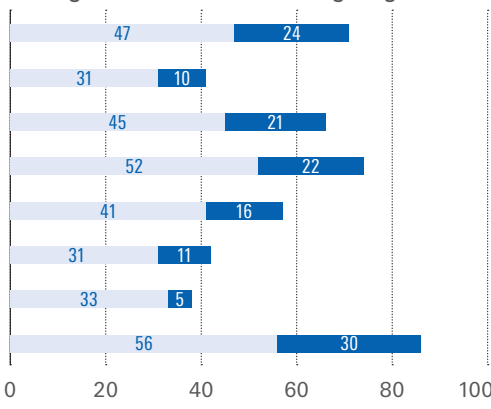

A Basis der Befragung sind 2057 Betriebe der Metall- und Elektro-, Holz- und Kunststoff- sowie Textil- und Bekleidungsindustrie

Trifft zu“: 173 Betriebe, "Trifft nicht zu“: 702 Betriebe

Die Antwortkategorien "Trifft eher nicht zu”, "Trifft teilweise zu" und "Lässt sich nicht beurteilen" sind hier nicht ausgewiesen. Deswegen addieren sich die Prozentwerte nicht auf 100 auf. Mir ging es um die Kontrastierung von Betrieben, die eindeutig über eine Strategie verfügen, mit denen, die eindeutig über keine Strategie verfügen

Quelle: Darstellung des Autors auf Basis der Daten des IG-Metall-Transformationsatlas, siehe Fußnote 2

Die Interessenvertretungen in den betrieblichen Transformationsprozess einzubeziehen bzw. diesen durch ihre Initiative überhaupt anzustoßen verspricht konkrete, für Betriebe und Beschäftigte lohnende Ergebnisse, denn: Neue Produkte sichern Arbeitsplätze. Es wird an Innovationen gearbeitet, und sie werden erprobt. Personaleinsatz und Qualifikation werden intensiver und genauer geplant. Eine kooperative Strategiearbeit ist jedoch nur dann auf Dauer möglich, wenn diese Voraussetzungen erfüllt sind: Betriebsleitungen müssen sich für eine Kooperation mit dem Betriebsrat öffnen, und Betriebsräte müssen ihre Vorbehalte gegenüber einem Co-Management überwinden. Sie brauchen dafür jedoch die explizite Zustimmung der Belegschaft. Noch eine weitere Voraussetzung muss gegeben sein: Es reicht nicht, Haltung und Einstellungen zu ändern. Die Betriebsräte müssen für diese neuen Herausforderungen qualifiziert und von erfahrenen gewerkschaftlichen Betriebsbetreuer*innen begleitet und betreut werden. Das gilt insbesondere für die Interessenvertretungen, für die das Thema der Entwicklung einer Betriebsstrategie Neuland ist. Und das ist die Mehrheit.

\section{AUTOR}

DETLEF GERST, Dr. disc. pol., ist Ressortleiter „Zukunft der Arbeit" beim Vorstand der IG Metall. Arbeitsschwerpunkte: Digitalisierung von Arbeit, Transformation, Agile Arbeit.

detlef.gerst@igmetall.de 